\title{
CABLE ASSISTED RENDEZVOUS FOR AIRCRAFT WITH SURFACE LOCATIONS
}

\author{
Daniel Sgarioto and Pavel Trivailo. \\ School of Aerospace, Mechanical and Manufacturing Engineering, RMIT University, \\ PO Box 71, Bundoora, Victoria, 3083, Australia. \\ S9908712@student.rmit.edu.au
}

\begin{abstract}
By systematically altering the in-plane flight path and velocity of an aircraft and the rate at which the cable is deployed, the tip of a cable being towed behind an aircraft is shown to rendezvous with a ground-based surface location in minimum time, with minimum control effort required. This is achieved through optimal control, formulated as a nonlinear programming problem using the Legendre-pseudospectral method. This method is fast, efficient and accurate. Results depicting a typical payload transportation operation are presented and discussed, along with directions for future research. The robustness of the method to disturbances is also explored. Copyright (C) 2005 IFAC
\end{abstract}

Keywords: Cables, optimal control, nonlinear programming, dynamic modelling.

\section{INTRODUCTION}

There are many exciting applications involving the use of a cable towed behind an aircraft. These include the delivery of food/medical supplies to remote communities (Russell and Anderson 1977), precise aerial bushfire fighting and search and rescue (Trivailo, et al. 2003, 2004), towing sonar devices (Etkin 1998) and charged cables (Trivailo, et al. 2004, 2003) for mine sweeping and environmental management using towed instrumentation (Etkin 1998). Hence one would expect research concerning the control of aerial tethered systems to be well established, yet this is not true and more research is needed for the aforementioned applications to fully materialize. Whilst some research has centred on the development of payload-based control laws to stabilize an airborne payload (Henderson, et al. 1999; Bourmistrov, et al. 1995; Cochran, et al. 1992), the development of cable-based control laws for more practical purposes (i.e. payload transportation) is limited. The concept of using an aerial tether to rendezvous with a surface location was successfully demonstrated by Trivailo, et al. (2004). This paper advances that work by utilizing a more representative cable model; studying a more practical rendezvous scenario and employing optimal feedback control for trajectory following to enhance robustness.

\section{AERIAL TOWED SYSTEM MODEL}

The model developed by the authors to accurately and efficiently simulate the in-plane dynamics of the aerial tethered system is shown in Figure 1.

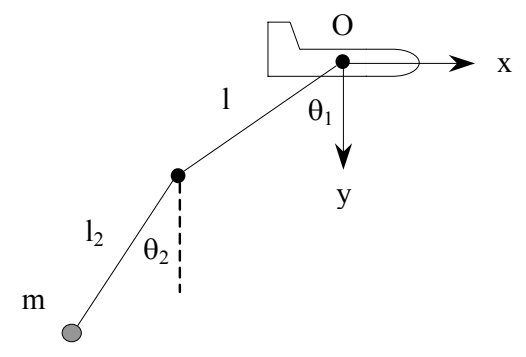

Fig. 1. Towed Aerial Cable-Body System Model.

It is assumed that the aircraft tows a homogeneous sphere of constant mass, drag coefficient and diameter via a rigid massive tether with a constant circular diameter. The aircraft is permitted to perform in-plane two-dimensional manoeuvres and has an infinite mass so it is unaffected by the dynamics of the cable/body combination. Table 1 shows the physical parameters that govern the model.

Table 1 Parameters governing the towed system model

\begin{tabular}{lll} 
Parameter & Description & Value \\
\hline $\mathrm{g}$ & Gravitational Constant & $9.81 \mathrm{~m} / \mathrm{s}^{2}$ \\
$\rho$ & Air Density & $1.23 \mathrm{~kg} / \mathrm{m}^{3}$ \\
$\rho_{\mathrm{C}}$ & Cable Mass Density & $4000 \mathrm{~kg} / \mathrm{m}^{3}$ \\
$\rho_{\mathrm{P}}$ & Payload Mass Density & $1000 \mathrm{~kg} / \mathrm{m}^{3}$ \\
$\mathrm{~d}_{\mathrm{C}}$ & Cable Diameter & $2.5 \mathrm{~mm}$ \\
$\mathrm{~d}_{\mathrm{P}}$ & Payload Diameter & $0.75 \mathrm{~m}$ \\
$\mathrm{l}_{2}$ & Lower Link Length & $500 \mathrm{~m}$ \\
$\mathrm{C}_{\mathrm{DC}}$ & Cable Drag Coefficient & 1.1 \\
$\mathrm{C}_{\mathrm{DP}}$ & Payload Drag Coefficient & 0.5 \\
\hline
\end{tabular}


The cable is discretized using two rigid links of equal diameter and similar material, however the upper link has variable length, whilst the lower link's length is fixed. The actuators used to control the dynamics of the system are the forward and vertical acceleration of the aircraft, along with the rate at which the deployment speed of the upper cable link changes with time. As for external forces, these were limited to aerodynamic and gravitational forces experienced by the cable and payload. Gravity forces acting on the cable were taken at the centre of each link and at the cable tip for the payload. Similarly, the aerodynamic force acting on the cable is proportional to the squared angular velocity seen by each cable link centre; proportional to the squared angular velocity at the tip for the payload. The areas projected by the cable links and the payload normal to the incoming flow were used for the aerodynamics.

By employing Kane's Equations, with the cable angles $\left\{\theta_{1}\right.$ ,$\left.\theta_{2}\right\}$, the non-dimensional length of the upper cable link $l$, and the non-dimensional range and altitude of the aircraft $\{x, y\}$ used as generalized coordinates, the non-dimensional equations of motion for the system may be written as:

$$
\begin{aligned}
& \ddot{x}=u_{1} \\
& \ddot{y}=u_{2} \\
& \ddot{\theta}=f_{1}\left(\ddot{x}, \ddot{y}, \ddot{l}, \dot{x}, \dot{y}, \dot{l}, \dot{\theta}_{1}, \dot{\theta}_{2}, \theta_{1}, \theta_{2}, l, t\right) \\
& \ddot{\theta}_{2}=f_{2}\left(\ddot{x}, \ddot{y}, \ddot{l}, \dot{x}, \dot{y}, \dot{l}, \dot{\theta}_{1}, \dot{\theta}_{2}, \theta_{1}, \theta_{2}, l, t\right) \\
& \ddot{l}=u_{3}
\end{aligned}
$$

where $u_{1}, u_{2}$ and $u_{3}$ are the chosen control variables for the system, while $f_{1}$ and $f_{2}$ are highly complex nonlinear expressions involving the system states and controls.

\section{CABLE ASSISTED RENDEZVOUS PROBLEM}

The rendezvous problem studied in this paper is illustrated in Figure 2. Depicting a payload delivery operation, a payload towed behind an aircraft via a cable is delivered to a location on the ground without landing the aircraft.

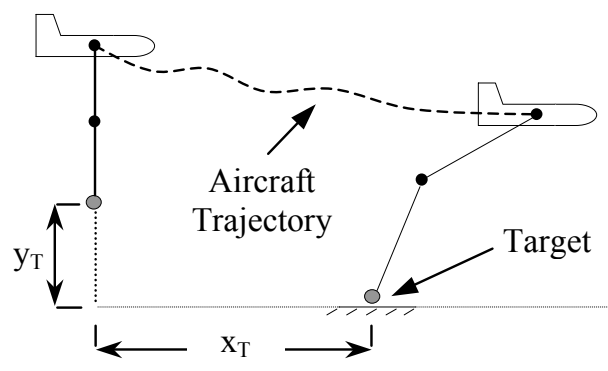

Fig. 2. Graphical Depiction of the Rendezvous Scenario.

The rendezvous manoeuvre begins with the aircraft in steady level flight, with the cable initially hanging vertically. The aircraft is then flown and the cable is deployed so as to rendezvous the tip of the cable with the location on the ground as quickly as possible. The aircraft is initially flying steadily at $65 \mathrm{~m} / \mathrm{s}$, at an altitude of 1500 $\mathrm{m}$, towing a $1000 \mathrm{~m}$ long cable. The target on the ground is initially $2000 \mathrm{~m}$ from the aircraft $\left(x_{\mathrm{T}}\right)$ and $500 \mathrm{~m}$ below the cable tip $\left(y_{\mathrm{T}}\right)$. Rendezvous is purely instantaneous.

\section{CONTROL SYSTEM DESIGN}

This section presents the control system design for the airborne towed system in order to successfully perform the in-plane rendezvous manoeuvre. In this paper, the twodimensional dynamics of the airborne tether are to be controlled by altering the forward and vertical acceleration of the aircraft, along with managing the rate at which the tether is being deployed/retrieved. Trivailo, et al. (2004) found that such a task was difficult due to the high drag forces the system experiences, along with the nonlinear coupling between the tether dynamics and control that reduced controllability. Similarly, the increased sophistication of the cable model employed here is expected to further complicate the proposed control undertaking.

The objective of the proposed control system is to determine an optimal trajectory for the aerial towed-cablebody system that directs the tip of the tether from a given initial state to rendezvous with a desired ground-based surface location. For the rendezvous case considered here, the trajectory that satisfies the boundary conditions, minimizes the difference between the desired and actual terminal states, minimizes the final time and the required control work will be considered the optimal solution.

\subsection{Optimal Control Theory}

Consider the problem of minimizing the performance index

$$
\begin{aligned}
J & =M\left[\ddot{x}\left(t_{f}\right), \dot{x}\left(t_{f}\right), x\left(t_{f}\right), t_{f}\right\rfloor \\
& +\int_{t_{0}}^{t_{f}}[L(\ddot{x}(t), \dot{x}(t), x(t), u(t), t) d t]
\end{aligned}
$$

where $t \in R, x \in R^{p}$ and $u \in R^{q}$ are subject to the dynamical constraints

$$
f[\ddot{x}(t), \dot{x}(t), x(t), u(t), t]=0, \quad t \in\left[t_{0}, t_{f}\right]
$$

and boundary conditions

$$
\begin{aligned}
& \varphi_{0}\left[\dot{x}\left(t_{0}\right), x\left(t_{0}\right), t_{0}\right]=0 \\
& \varphi_{f}\left[\dot{x}\left(t_{f}\right), x\left(t_{f}\right), t_{f}\right]=0
\end{aligned}
$$


where $\varphi_{0} \in R^{r}$ and $\varphi_{f} \in R^{s}$ with $r \leq p$ and $s \leq p$, and the state and control constraints

$$
g[\dot{x}(t), x(t), u(t)] \leq 0, \quad g \in R^{v}
$$

Betts (1998) has identified a host of methods to solve optimal control problems governed by (6) through to (9). These fall broadly within two groups; indirect methods (Betts 1998; Ohtsuka and Fujii 1994; Kirk 1970) and direct methods (Trivailo, et al. 2004; Williams 2003; Blanksby, et al. 2003; Fahroo and Ross 2001, 2002; Hull 1997; Elnagar, et al. 1995; Enright and Conway 1992; Hargraves and Paris 1987). Indirect methods involve using the calculus of variations to determine the optimality conditions and require solving the highly unstable co-state equations, making these methods particularly unattractive. However direct methods circumvent these difficulties by transforming the continuous optimal control problem into a more manageable discrete parameter optimization problem. In this paper, a direct method known as the Legendre pseudospectral method was chosen to solve the optimal control problem that governs the desired rendezvous problem. This method is relatively easy to implement and enjoys spectral accuracy and efficient computation times (Fahroo and Ross 2002; Elnagar, et al. 1995).

\subsection{The Legendre Pseudospectral Method}

This section briefly outlines the application of the Legendre-pseudospectral method to optimal control problems. Readers are referred to Williams (2003), Blanksby, et al. (2003) and Elnagar, et al. (1995) for details of the actual discretization process. The essence of the method is to interpolate the state and control trajectories using Legendre polynomials. Legendre polynomials belong to a class of globally orthogonal Jacobi polynomials. The grid points associated with the discretization are chosen as the roots of the interpolating Legendre polynomials, known as Legendre-Gauss-Lobatto points. The state equations are enforced as dynamic constraints by differentiating the approximating Legendre polynomials at the corresponding Gauss-Lobatto points. Hence, the time consuming task of integrating the state equations is avoided. The performance index is approximated using an appropriate numerical quadrature procedure; Gauss-Lobatto quadrature was employed in this paper. Once the original optimal control problem is discretized and formulated as a nonlinear programming problem, the structure of the discretized problem resembles that of the original continuous problem.

\subsection{Application to the Cable Assisted Rendezvous Problem}

The optimal control problem that characterizes the rendezvous problem is formulated as follows:
Find the control inputs to minimize

$$
\begin{aligned}
& J=\left(x_{T I P}-x_{T I P f}\right)^{2}+\left(y_{T I P}-y_{T I P f}\right)^{2} \\
& +\left(\dot{\theta}_{1}-\dot{\theta}_{1 f}\right)^{2}+\left(\dot{\theta}_{2}-\dot{\theta}_{2 f}\right)^{2}+\left(i-\dot{l}_{f}\right)^{2}+t_{f} \\
& +1 / 2 \int_{0}^{t_{f}} u_{1}{ }^{2}+u_{2}{ }^{2}+u_{3}{ }^{2} d t
\end{aligned}
$$

subject to the dynamical constraints given by (1) to (5) and

$$
\begin{aligned}
& \left\{x, y, \theta_{1}, \theta_{2}, l, \dot{x}, \dot{y}, \dot{\theta}_{1}, \dot{\theta}_{2}, i\right\}_{t=t_{0}}= \\
& \left\{0,0,0^{\circ}, 0^{\circ}, 500,65,0,0,0,0\right\} \\
& \left\{\dot{\theta}_{1}, \dot{\theta}_{2}, i\right\}_{t=t_{f}}=\{0,0,0\}
\end{aligned}
$$

and the control inequalities

$$
\begin{aligned}
& -10 \leq u_{1}(t) \leq 10 \\
& -7.5 \leq u_{2}(t) \leq 7.5,\left[\mathrm{~m} / \mathrm{s}^{2}\right] \\
& -7.5 \leq u_{3}(t) \leq 7.5
\end{aligned}
$$

DIRECT (Williams 2004), a MATLAB ${ }^{\circledR}$ based application for solving optimal control, dynamic optimization and parameter estimation problems, was utilized to solve the nonlinear programming problem that represents the rendezvous problem given by (10) through to (12). DIRECT utilizes SNOPT (Gill, et al. 2002) as the nonlinear solver, implemented in MATLAB $^{\circledR}$ via mex files. DIRECT has an efficient pattern generator for the Jacobian of the constraints; hence analytical Jacobians were not provided.

The initial guess of the optimization variables for largescale nonlinear optimal control problems is important. Generally it is not known a priori if a solution exists for a given problem, so to improve the efficiency and expediency of the optimization process, a good quality guess for the solution should be provided to the solver. Not only does this reduce solver workload, often a solution may not be found if a poor initial guess is provided. In this paper, the initial guess is generated by first numerically integrating the state equations with no applied controls, then interpolating the appropriate values for the states at the corresponding grid points. The optimization process commences with the level of discretization $N$ set at a modest value and a solution to the problem is found using the aforementioned initial guess. The level of discretization is subsequently increased, with the previous solution used as a new improved guess. This iterative process continues until the solution converges; the computational cost of further increasing the discretization level begins to outweigh any potential improvements to the accuracy of the solution. 


\subsection{Neighbouring Optimal Feedback Control}

In order to ascertain how sensitive the proposed non-linear optimal control scheme is to external disturbances, an optimal feedback controller was designed for the aerial towed cable system. The method chosen to implement this was Linear Quadratic Regulator (LQR) control. This method determines the optimal feedback gain matrix $K$, such that the following state feedback control law

$u(t)=-K x$

minimises the cost function

$J=\int_{0}^{\infty}\left(x^{T} Q x+u^{T} R u\right) d t$

subject to the linearized state equations

$\dot{x}=A(t) x+B(t) u$

where $A$ and $B$ are the time varying state and control influence matrices, $Q$ and $R$ are symmetric positive semi definite and symmetric positive definite design matrices respectively. By carefully choosing values for $Q$ and $R$, it is possible to ensure that the system is able to recover from deviations about the nominal optimal trajectory, assuming that such deviations are not excessive. In this paper, $R$ was selected as the identity matrix [I], while $Q$ was chosen to be $10[I]$. The influence matrices $A$ and $B$ system are obtained by linearizing (1) to (5) about the nominal trajectory.

Time varying wind gusts, modelled as full sine functions, were chosen as the external disturbances for the system. The system encounters two gusts, the first having horizontal and vertical components of -2.5 and $3.75 \mathrm{~m} / \mathrm{s}$ respectively, the second having velocity components of 1.5 and $-3 \mathrm{~m} / \mathrm{s}$ respectively. The first gust occurs 2.5 secs into the manoeuvre and lasts for $7.5 \mathrm{secs}$; the final gust occurs after 15 secs and lasts for 5 secs.

\section{RESULTS}

\subsection{Optimization Results}

The results of the optimization task are the focus of this section. The optimization process began at $N=30$, with the level of discretization subsequently increased by intervals of 20 until the final discretization level of 90 was reached, which represented a compromise between execution time and accuracy. The time required for the optimization on a Pentium 4 processor was 4314.89 secs, which is excellent given the size and complexity of the parameter optimization problem at hand. The final value of the performance index $J_{f}$ returned by DIRECT was 3.0390037. This corresponds to rendezvous occurring after 32.78 secs of manoeuvring. Examination of the exit flag and constraint file returned by SNOPT indicated the optimization was successful; the optimal solution was found with all target states being met.

\subsection{Numerical Results}

The results found by applying both open and closed loop optimal control to the aerial towed system are shown in Figure 3 through to Figure 9. Figure 3 and Figure 4 detail the horizontal and vertical paths the aircraft follows for successful rendezvous, along with the respective velocity time histories. Figure 5 and Figure 6 show the angular displacement and velocity of the upper and lower cable links respectively, while Figure 7 presents the radial displacement and velocity for the upper cable link. Figure 8 depicts the aircraft forward and vertical acceleration, along with the upper cable link radial acceleration. Although not shown, there is an excellent agreement between the discrete Legendre-pseuodspectral approximation and the continuous solution generated by applying the discrete controls (found from DIRECT) to the continuous state equations; only a maximum difference of $1.04 \%$ exists between the two. Finally, Figure 9 shows the cable tip trajectory during the rendezvous manoeuvres. In all figures, the solid line denotes the closed loop trajectories; the solid marker depicts the optimal nominal results without gusts, while the broken line signifies the open loop responses with gusts applied.
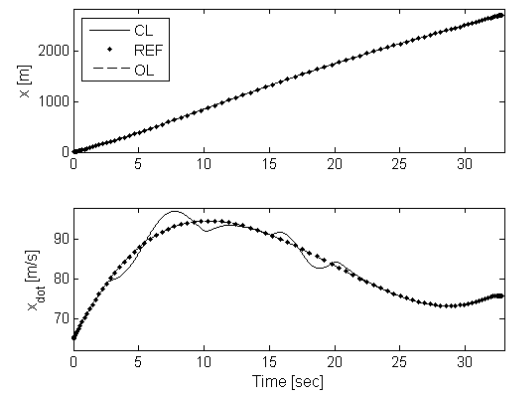

Fig. 3. Trajectory for Rendezvous, (a) Aircraft Horizontal Displacement; (b) Aircraft Forward Velocity.
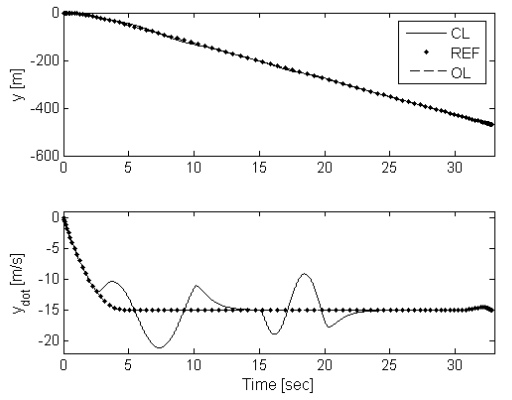

Fig. 4. Trajectory for Rendezvous, (a) Aircraft Vertical Displacement; (b) Aircraft Vertical Velocity. 

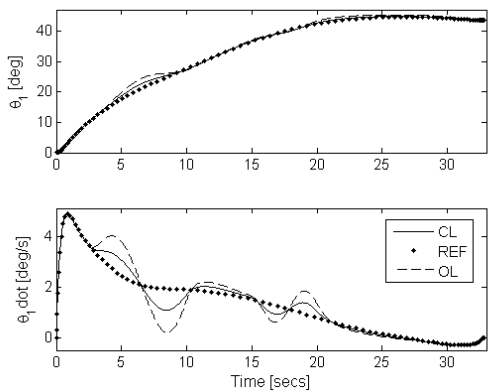

Fig. 5. Trajectory for Rendezvous, (a) Upper Cable Link Displacement; (b) Upper Cable Link Velocity.
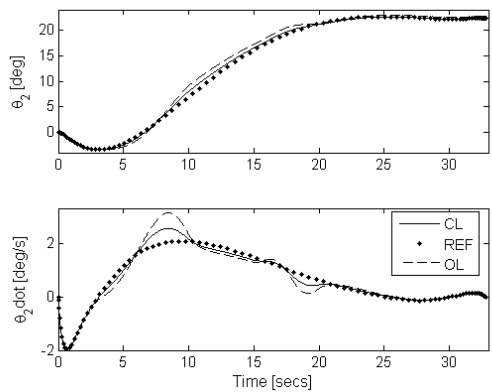

Fig. 6. Trajectory for Rendezvous, (a) Lower Cable Link Displacement; (b) Lower Cable Link Velocity.
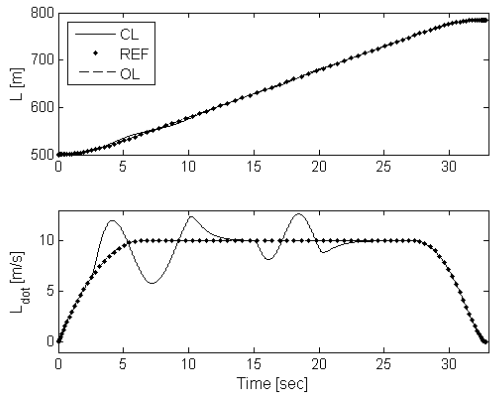

Fig. 7. Trajectory for Rendezvous, (a) Upper Cable Link Length; (b) Upper Cable Link Radial Velocity.
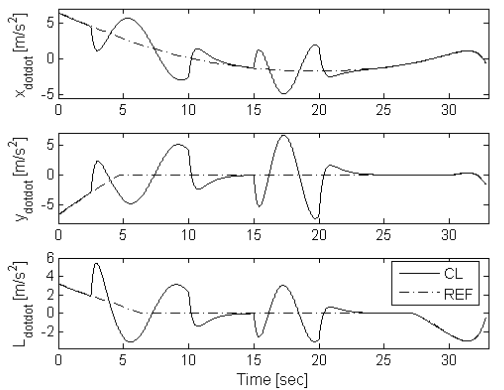

Fig. 8. Control for Rendezvous, (a) Aircraft Forward Acceleration; (b) Aircraft Vertical Acceleration; (c) Upper Link Radial Acceleration.

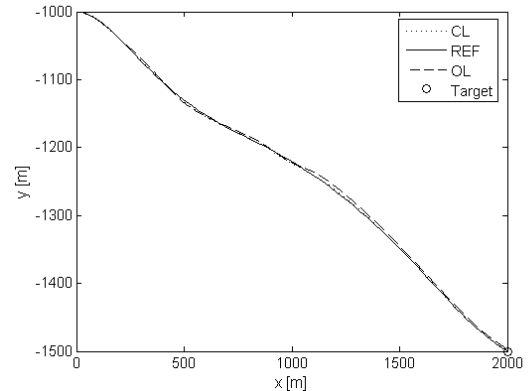

Fig. 9. Optimal Cable Tip Trajectory for Rendezvous

\section{DISCUSSION}

Figure 3 and Figure 4 show that to minimize the time for rendezvous, the aircraft initially dives as quickly as possible towards the target, begins to pull up mid-flight, before quickly diving again during the latter stages of the flight. Depending on the nature of the gusts, it can be seen from Figure 3 and Figure 4 that the feedback controller significantly alters the aircraft's speed so as to compensate for an increase/decrease in velocity the aircraft encounters due to the gusts. As evidenced by Figure 5 and Figure 6, the cable initially swings about slowly, before damping out towards the end of the manoeuvre. As expected, the cable swings more freely and further over the duration of the gusts, but once the gusts have terminated the cable returns quickly to its optimal state, even without the corrective measures provided by the feedback. The inherently large drag forces the cable experiences tend to quickly damp out the oscillations the gusts cause, suggesting that the cable itself is not particularly sensitive to the gusts. However, feedback control ensures that the oscillations are much smaller, lessening their impact on the system. As the aircraft appears to be more sensitive to gust activity than the cable, applying feedback control directly to the aircraft and not the cable would be more appropriate. The deployment profile given in Figure 7 reveals that initially the cable should be deployed quickly and maintained that way for most of the manoeuvre, before being retrieved quickly late in the rendezvous attempt. Additional deployment and retrieval is needed over the duration of the gusts to help nullify their effect on the system's dynamics. In Figure 8, the amount of control needed to perform the rendezvous manoeuvre is modest, focused mainly at the start and end of the manoeuvre. Intuitively, as the gusts are encountered, more control effort is required to return the system to its nominal trajectory, although such increases are acceptable. Even when gusts are encountered, Figure 9 shows the cable tip following a smooth trajectory during the rendezvous manoeuvres. Without feedback, the gusts render the cable tip $8.63 \mathrm{~m}$ in front of the rendezvous point, whilst feedback reduces this error to $1.82 \mathrm{~m}$. The use of receding horizon control with a final state penalty term in the cost function should reduce this error even further. 


\section{FURTHER WORK}

While the results presented in this paper are encouraging, more research is required to further develop this field. The areas of robustness, modelling and the concept of payload capture/delivery are requisite future research domains.

\section{CONCLUSIONS}

It was successfully demonstrated that a payload at the end of a cable being towed by an aircraft could rendezvous with a surface location in minimum time, whilst expending minimum control effort, by manipulating the aircraft's acceleration and the rate at which the cable is being deployed. This was achieved by formulating an optimal control problem, discretized using the Legendrepseudospectral method, and solved using the nonlinear solver SNOPT implemented in MATLAB. Such an approach was easily implemented, fast and very accurate. Preliminary results generated for a typical small-scale payload transportation operation are encouraging. For minimum rendezvous time, the aircraft initially dives as quickly as possible towards the target, pulls up mid-flight, before quickly diving again during the latter stages of the flight. Initially, the cable is deployed quickly, maintained that way for most of the manoeuvre, before being swiftly retrieved late in the rendezvous attempt. The cable tip trajectories were very smooth during the rendezvous manoeuvres even as gusts were encountered, although LQR control was successfully applied to improve robustness.

\section{ACKNOWLEDGEMENTS}

This work is supported by the School of Aerospace, Mechanical and Manufacturing Engineering, RMIT University, Australia. The support given by Paul Williams in providing the use of DIRECT is also duly acknowledged.

\section{REFERENCES}

Betts, J.T. (1998). Survey of numerical methods for trajectory optimization. Journal of Guidance, Control and Dynamics, 21(2), 193-207.

Blanksby, C.M., P. Williams and P.M. Trivailo (2003). Tether assisted rendezvous for satellites with small relative inclinations. In: Proceedings of the $54^{\text {th }}$ International Astronautical Congress, Bremen, Germany, pp. 1-11.

Bourmistrov, A.S., R.D. Hill and P. Riseborough (1995). Nonlinear control law for aerial towed target. Journal of Guidance, Control and Dynamics, 18(6), 1232-1238.

Cochran, J.E., M. Innocenti, T.S. No and A. Thurkal (1992). Dynamics and control of maneuverable towed flight vehicles. Journal of Guidance, Control and Dynamics, 15(5), 1245-1252.
Elnagar, G., M. A. Kazemi and M. Razzaghi (1995). The pseudospectral legendre method for discretizing optimal control problems. IEEE Transactions on Automatic Control, 40(10), 1793-1796.

Enright, P.J. and B.A. Conway (1992). Discrete approximations to optimal trajectories using direct transcription and nonlinear programming. Journal of Guidance, Control and Dynamics, 15(4), 994-1002.

Etkin, B. (1998). Stability of a towed body. Journal of Aircraft, 35(2), 197-205.

Fahroo, F. and I.M. Ross (2002). Direct trajectory optimisation by a chebyshev pseudospectral method. Journal of Guidance, Control and Dynamics, 25(1), 160-166.

Fahroo, F. and I.M. Ross (2001). Costate estimation by a Legendre pseudospectral method. Journal of Guidance, Control and Dynamics, 24(2), 270-277.

Gill, P.E., W. Murray and M.A. Saunders (2002). Users Guide to SNOPT Version 6: A Fortran Package for Large-Scale Nonlinear Programming. Report NA 02-2, Department of Mathematics, University of California, San Diego, USA.

Hargraves, C.R. and S.W. Paris (1987). Direct trajectory optimisation using nonlinear programming and collocation. Journal of Guidance, Control and Dynamics, 10(4), 338-324.

Henderson, J.F., J. Potjewyd and B. Ireland (1999). The dynamics of an airborne towed target system with active control. In: Proceedings of the Institution of Mechanical Engineers, Journal of Aerospace Engineering, Vol. 213, Part G, pp. 305-319.

Hull, D.G. (1997). Conversion of optimal control problems into parameter optimisation problems. Journal of Guidance, Control and Dynamics, 20(1), 994-1002.

Kirk, D.E. (1970). Optimal control theory: an introduction. Chap. 6, pp. 329-409, Prentice Hall Inc., New Jersey, USA.

Ohtsuka, T. and H. A. Fujii (1994). Stabilized continuation method for solving optimal control problems. Journal of Guidance, Control and Dynamics, 17(5), 950-957.

Russell, J.J. and W.J. Anderson (1977). Equilibrium and stability of a circularly towed cable subject to aerodynamic drag. Journal of Aircraft, 14(7), 680-686.

Trivailo, P.M., C.M. Blanksby, D.E. Sgarioto, P. Williams and R. Smart (2003). Defence applications for cable systems deployed from aerial and naval platforms. In: Proceedings of the Land Warfare Conference 2003 Conference, Adelaide, Australia, pp. 395-403.

Trivailo, P.M., D.E. Sgarioto and C.M. Blanksby (2004). Optimal control of aerial tethers for payload rendezvous. In: Proceedings of the $5^{\text {th }}$ Asian Control Conference, Melbourne, Australia, pp. 285-294.

Williams, P. (2004). Users Guide to DIRECT Version 1.11. unpublished paper, pp. 1-10.

Williams, P. (2003). Jacobi pseudospectral method for solving optimal control problems. Journal of Guidance, Control and Dynamics, 27(2), 293-297. 